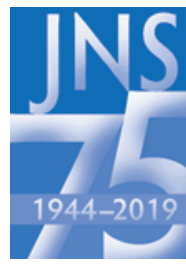

\title{
Prospective multicenter studies in pediatric hydrocephalus
}

\author{
JNSPG 75th Anniversary Invited Review Article \\ John R. W. Kestle, MD, ${ }^{1}$ and Jay Riva-Cambrin, MD, MSc ${ }^{2}$ \\ 1Department of Neurosurgery, Division of Pediatric Neurosurgery, Primary Children's Hospital, University of Utah, Salt Lake City, \\ Utah; and 2Department of Clinical Neurosciences, University of Calgary, Alberta, Canada
}

\begin{abstract}
Prospective multicenter clinical research studies in pediatric hydrocephalus are relatively rare. They cover a broad spectrum of hydrocephalus topics, including management of intraventricular hemorrhage in premature infants, shunt techniques and equipment, shunt outcomes, endoscopic treatment of hydrocephalus, and prevention and treatment of infection. The research methodologies include randomized trials, cohort studies, and registry-based studies. This review describes prospective multicenter studies in pediatric hydrocephalus since 1990. Many studies have included all forms of hydrocephalus and used device or procedure failure as the primary outcome. Although such studies have yielded useful findings, they might miss important treatment effects in specific subgroups. As multicenter study networks grow, larger patient numbers will allow studies with more focused entry criteria based on known and evolving prognostic factors. In addition, increased use of patient-centered outcomes such as neurodevelopmental assessment and quality of life should be measured and emphasized in study results. Well-planned multicenter clinical studies can significantly affect the care of children with hydrocephalus and will continue to have an important role in improving care for these children and their families.
\end{abstract}

https://thejns.org/doi/abs/10.3171/2018.10.PEDS18328

KEYWORDS pediatric hydrocephalus; randomized controlled trial; cohort study; prospective; multicenter

$\mathrm{T}$ HE current emphasis on evidence for clinical decision-making was predicted in 1953 by one of the forefathers of pediatric neurosurgery, Don Matson. ${ }^{26}$ In his landmark paper, "Hydrocephalus treated by arachnoid-ureterostomy," he described a variety of surgical procedures for managing progressive hydrocephalus. $\mathrm{He}$ advised that "An objective analysis of longer term results of such operations with respect to the growth and development of these children over a period of years is needed-not in a single isolated patient, but in a group of patients treated by similar technique." Not only did he describe the need for careful assessment of different procedures for hydrocephalus, but also more than 60 years ago he suggested that the results should be assessed with respect to the growth and development of the children. For many years, hydrocephalus research has focused on procedural failure, but recent clinical trials have made the important decision to include developmental outcome in the primary analysis. ${ }^{21,44}$
In this review, we focus on studies that involved prospective data collection across multiple centers. A number of clinical research designs that incorporate prospective multicenter collaboration, including randomized controlled trials (RCTs), nonrandomized prospective studies, and registries, have all been used to study pediatric hydrocephalus.

\section{Prospective Multicenter Clinical Research}

Multicenter clinical studies have a number of advantages and challenges. One of the most significant advantages is the ability to accumulate adequate sample size in a reasonable time frame. It is often the sample size calculation that leads to the need for multiple centers. The inclusion of multiple centers also offers a chance to identify variation in practice and results among sites. This allows for adjustment of the analysis to account for center differences. Variation among centers may identify center-specific tech-

ABBREVIATIONS AIC = antibiotic-impregnated catheter; BASICS = British Antibiotic- and Silver-Impregnated Catheters Study; CDP = Core Data Project; CI = confidence interval; $\mathrm{CPC}=$ choroid plexus cauterization; DRIFT = drainage, irrigation, and fibrinolytic therapy; ESIT = Endoscopic Shunt Insertion Trial; ETV = endoscopic third ventriculostomy; HCRN = Hydrocephalus Clinical Research Network; HR = hazard ratio; IVH = intraventricular hemorrhage; OSV = Orbis Sigma Valve; PHVD = posthemorrhagic ventricular dilation; RCT = randomized controlled trial; SDT = Shunt Design Trial; sIRB = single IRB.

SUBMITTED October 3, 2018. ACCEPTED October 9, 2018.

INCLUDE WHEN CITING DOI: 10.3171/2018.10.PEDS18328. 
niques that are efficacious and can be incorporated across other sites or assessed in future research.

The prospective nature of the work allows definition of specific data elements required to answer the question so they can be intentionally captured rather than collecting only what might happen to be in the medical record (as in a retrospective study). Adequate data are sought to answer the study question, making the database more complete and accurate. Furthermore, prospective studies often have mechanisms for identifying and retrieving missing data close in time to the clinical event when the details are more accessible.

Among the challenges facing the investigators of a multicenter study are the organization and institutional ethics approval required. Organizing a prospective multicenter clinical study requires collaboration among clinicians, clinical research experts, data managers, and biostatisticians. The participants must agree on a study question and protocol, which will include the specific condition being studied (entry criteria), the intervention of interest, and the primary outcome. ${ }^{31}$

Prospective multicenter studies require IRB approval, which can be accomplished in a couple of ways. Usually, the lead clinical site submits an application to the local IRB. Once approved, the protocol and the lead site's IRB approval are submitted to each site's IRB. It is very important that any modifications to the protocol be made across the entire study group. Recently, a "single IRB (sIRB)" process is becoming more common and is now required for NIH multicenter clinical trials. ${ }^{27}$ This is a process in which one IRB acts as the sIRB for the group. After a process of negotiation, all other site IRBs agree to accept the decision of the sIRB. Study approval and revision then go through the sIRB on behalf of all sites.

\section{Multicenter Prospective RCTs in Pediatric Hydrocephalus}

Since 1990, 10 multicenter RCTs in pediatric hydrocephalus have been completed and 2 are in progress and have finished patient accrual. These studies include 3740 children from 189 centers. Four of the studies ${ }^{4,12,30,44}$ addressed questions related to the management of premature children with intraventricular hemorrhage (IVH), 2 studies $^{7,29}$ compared different shunt valves, 2 studies ${ }^{14,41}$ assessed surgical techniques used during shunt placement, 2 studies ${ }^{21,22}$ compared endoscopic and shunt treatments, 1 study compared the incidence of hydrocephalus after fetal or postnatal closure of myelomeningocele, ${ }^{1}$ and 1 trial compared the ability of three types of shunt catheters to reduce infections. ${ }^{11}$ Only 2 of the 10 completed studies found a significant difference in the primary outcome; ${ }^{1,44}$ the other 8 found no difference between treatment arms.

\section{Studies Related to the Management of Premature Children With IVH}

Four RCTs have studied premature children with posthemorrhagic ventricular dilation (PHVD). The goal of 3 studies was to improve patient survival and reduce shunt placement. One trial incorporated developmental assessment into the primary outcome.
The first two trials assessed interventions used by neonatologists to prevent hydrocephalus. In 1 trial, ${ }^{30}$ the authors compared early lumbar puncture performed in response to ventricular enlargement on ultrasonography with lumbar puncture performed in response to symptoms and increasing head circumference. The RCT demonstrated no reduction in the need for subsequent shunt insertion. Another neonatology study ${ }^{12}$ found no survival advantage to the addition of furosemide and acetazolamide over standard care; furthermore, the intervention arm had higher neurological morbidity than the control arm.

The first assessment of a surgical intervention for PHVD involved a procedure called drainage, irrigation, and fibrinolytic therapy (DRIFT). This procedure was compared with standard treatment in 77 children at 4 sites. ${ }^{43,44}$ DRIFT was a challenging treatment requiring two external ventricular drains and measurement of intracranial pressure. The ventricular system was injected with recombinant tissue plasminogen activator and then flushed with artificial CSF for 2-7 days. Patient accrual to the study stopped early because two patients in the treatment group had rebleeds. Despite those rebleeds, the treatment group had a significantly lower incidence of death or severe disability at the 2-year follow-up. This benefit persisted at the 10-year follow-up (Luyt K, Jary S, Lea $\mathrm{C}$, et al: Drainage Irrigation and Fibrinolytic Therapy [DRIFT] for post haemorrhagic ventricular dilatation: improved cognitive ability at school-age, presented at the Pediatric Academic Societies 2017 Meeting, San Francisco, CA, May 6-9, 2017). Despite these results, DRIFT has not become widely accepted, perhaps because of the logistics of placing two drains and infusing fluid for several days in a severely premature baby. Instead, a number of surgeons have taken the DRIFT results as proof of principle that clot removal is helpful. Reports have now emerged of clot removal/ventricular washout ("endoscopic ventricular lavage") at the time of surgery for reservoir placement. ${ }^{8,34}$ None of those reports have significant sample sizes, nor are they randomized, but the approach is intriguing and may deserve further investigation.

A recent study focused on the timing of intervention, with 126 infants at 14 sites randomized to early versus late intervention. ${ }^{5}$ In both study arms, a stepwise protocol of lumbar punctures, reservoir placement, then shunt insertion was followed. No difference was observed in patient survival or the need for shunting between the two treatment arms. Despite the lack of difference between the randomized arms, the overall incidence of shunting in this study was the lowest ever reported (21\%). This overall low shunting rate may be due to the very early treatment approach that was used. Even patients in the late group were treated earlier than is common practice in North America. It is therefore possible that the study cohort included some patients with milder disease who, if left untreated, would not need shunt placement. Of course, it may also be that earlier treatment is efficacious. Further study of the appropriate timing of intervention appears to be warranted.

\section{Studies Assessing the Performance of Shunt Valves}

Two trials in the late 1990s compared the time to failure of different shunt valves. In 1998, Drake et al. ${ }^{7}$ ran- 
domized patients receiving their first shunt to a Delta valve, an Orbis Sigma Valve (OSV), or a differential pressure valve. There was no significant difference in the time to shunt failure, ${ }^{7,13}$ a finding that has led some readers to the conclusion that "valves are all the same." This is an oversimplification, because many other valves on the market have not been assessed in this way (but should be). The appropriate conclusion of the trial is that there was not a $50 \%$ reduction in the 1-year failure rate with any of the valves tested.

In 1999, Pollack et al..$^{29}$ reported a trial in which an adjustable valve (Codman Hakim programmable) or a nonadjustable valve was implanted at the time of first shunt insertion or revision. Children and adults were included, and overall there was no difference in the time to shunt failure. In addition, there was no difference between treatment arms when insertions and revisions were considered separately. Two-thirds of programmable valves were adjusted after implantation, but there were no specific instructions given or criteria for making adjustments.

These two results are disappointing, but this is an area that should be studied further. Shunt failure is common enough that sample sizes are readily achievable. In addition, the biggest risk factor for infection is shunt revision. ${ }^{35}$ It therefore appears that studies to reduce shunt failure are feasible and meaningful and deserve more attention.

\section{Studies of Surgical Technique}

Two prospective multicenter RCTs have studied specific intraoperative techniques for shunt insertion. A trial comparing entry sites for the ventricular catheter is underway (Whitehead W: Rationale and methodology of the CSF Shunt Entry Site Trial, presented at the 7th Annual Meeting of the International Society for Hydrocephalus and Cerebrospinal Fluid Disorders, Banff, Alberta, Canada, September 19-21, 2015). Children receiving their first shunt are randomized to an anterior or posterior entry site with an intraventricular target of the frontal or occipital horn. Data pooled from several previous trials suggested an advantage to anterior shunt entry site ${ }^{41}$ even when the analysis was limited to catheters with the tip in the frontal horn. On the other hand, some studies showed no difference and/or an advantage to a posterior entry site..$^{2,3,6,23-25}$ The ongoing RCT, which uses time to shunt failure as the primary outcome, is approaching the required accrual of 448 children, and results are anticipated in about 2 years.

A multicenter randomized trial, reported in $2003,{ }^{14}$ randomized 393 children to insertion of the ventricular catheter with or without an endoscope. There was no difference in the time to shunt failure in the two groups. A secondary analysis based on catheter position suggested that catheters ending up away from the choroid plexus had better survival. The principle of avoiding the choroid plexus seems logical, and other studies have attempted to do so with ultrasonography ${ }^{42}$ or stereotaxy. ${ }^{10}$

\section{Studies Comparing Endoscopic Treatment of Hydrocephalus to Shunt Insertion}

The International Infant Hydrocephalus Study ${ }^{22}$ was a prospective multicenter study comparing endoscopic third ventriculostomy (ETV) with shunt insertion for children with aqueductal stenosis; this trial included a randomized arm and a nonrandomized arm. Children were entered into the randomized arm (ETV vs shunt) if their families were willing, and others entered a prospective observational cohort treated per family wishes. The trial included 27 centers, but accrual to the randomized arm was difficult. After 8 years, accrual was stopped, and the randomized $(n=52)$ and nonrandomized $(n=106)$ arms were pooled. The success rate (no need for repeat intervention for CSF diversion) at 12 months was $66 \%$ for ETV and $83 \%$ for shunts. The complete evaluation of 5-year outcomes in 78 children showed no difference in Health Utilities Index-2 score (mean $0.90 \pm 0.19$ for ETV, $0.94 \pm 0.10$ for shunt; $p$ $=0.21$ ) or 5-year Hydrocephalus Outcome Questionnaire overall score (mean $0.81 \pm 0.15$ for ETV, $0.85 \pm 0.12$ for shunt; $\mathrm{p}=0.42) .{ }^{22}$

Although we set out to describe the prospective multicenter studies, it is hard to ignore the recently reported randomized trial from Uganda. ${ }^{21}$ Infants with postinfectious hydrocephalus were randomized to ETV with choroid plexus cauterization (ETV+CPC) or shunt placement. The primary outcome was developmental assessment measured by Bayley Scales of Infant Development-III. The developmental scores in both groups were low, but neither procedure resulted in a significant developmental advantage. The study also measured brain and CSF volume. Although the ventricles were larger in the ETV+CPC group, the brain volume was the same as in the shunt patients. This landmark trial suggests that larger ventricles after ETV+CPC in this population do not harm development, at least in the 1st year. Further follow-up and similar research in noninfectious hydrocephalus will be very important.

\section{Fetal Closure of Myelomeningocele}

Fetal surgery for myelomeningocele significantly reduced the need for shunt placement compared with postnatal closure in an RCT reported in 2011. ${ }^{1}$ Subsequent analysis has indicated that fetal ventricle size can predict the response to fetal surgery and that fetuses with severe ventriculomegaly (ventricle size $>15$ ) do not appear to have a reduced risk of shunting. ${ }^{37}$

\section{BASICS (British Antibiotic- and Silver-Impregnated Catheters Study)}

The BASICS trial ${ }^{11}$ has just completed follow-up of more than 1200 patients from 17 sites in the UK and Ireland who were randomized among 3 different catheters (standard, antibiotic-impregnated [AICs], or silver-impregnated) at the time of shunt placement. Data analysis is underway, and results should be available in 2019.

\section{Nonrandomized, Multicenter Prospective Observational Studies in Pediatric Hydrocephalus}

Although RCTs are recognized as the highest level of medical evidence, in many circumstances they are not feasible because of a lack of clinical equipoise, rare outcomes 
requiring a very large sample size, prohibitive expense, and/or barriers to randomization, such as strong patient preference for one treatment. ${ }^{22}$ Thus, well-designed prospective cohort studies are substituted for RCTs, often with compelling results, and represent the next best level of evidence. When conducted across multiple sites, these observational studies allow large sample sizes, have good generalizability, and are usually more feasible than RCTs. This is the primary reason the conclusion sections of most retrospective single-center studies end with a statement indicating that "further investigation within a prospective, multicenter cohort study or RCT is required to validate our findings."

Despite these self-evident axioms, these sophisticated study types are rare (Fig. 1). Since 1990, there have been more than 7500 published papers on pediatric hydrocephalus, of which $7 \%$ are prospective, $2 \%$ are multicenter, $0.3 \%$ are multicenter prospective cohort studies, and $0.1 \%$ are RCTs. These numbers reflect the expense and the enormous organization required to conduct these types of studies.

Prospective observational studies may be part of a large registry or they may be designed as "standalone" cohort studies. The standalone studies have a clear study question, sample size, and timeline and a database designed to answer that specific question. In contrast, registries are usually open ended, without a planned sample size. They prospectively collect information that can be used to answer a number of questions, some of which may be planned upfront and others that arise later. Registries are also very helpful for tracking disease incidence and prevalence and monitoring trends in care over time (Tamber M, Kestle J, Browd S, et al: Temporal trends in surgical procedures for pediatric hydrocephalus - an analysis of the HCRN core data, presented at the AANS/CNS Section on Pediatric Neurosurgery 47th Annual Meeting, Nashville, TN, December 6-9, 2018). Both approaches have been used to study pediatric hydrocephalus.

\section{Prospective Multicenter Cohort Studies in Pediatric Hydrocephalus}

Before 2006, there was a paucity of these types of studies; however, with increased awareness of the principles of clinical epidemiology as well as the creation of the Hydrocephalus Clinical Research Network (HCRN), these study types are becoming more common now.

\section{Temporization Procedures in Preterm IVH}

A recent HCRN cohort study ${ }^{39}$ prospectively followed 145 preterm infants with grade III or IV IVH at 6 centers. A protocol with standard rubrics for decision-making was followed. Shunting and complication rates were the same in patients temporized by ventricular reservoir and those with ventriculosubgaleal shunts (no differences in infection $[p=0.71]$ or permanent shunting rates $[p=0.36]$ between the cohorts). This study highlights the importance of the prospective cohort study with a standard protocol for patient management, as a previous retrospective HCRN study had concluded that ventricular reservoirs resulted in fewer shunts than ventriculosubgaleal shunts. ${ }^{40}$

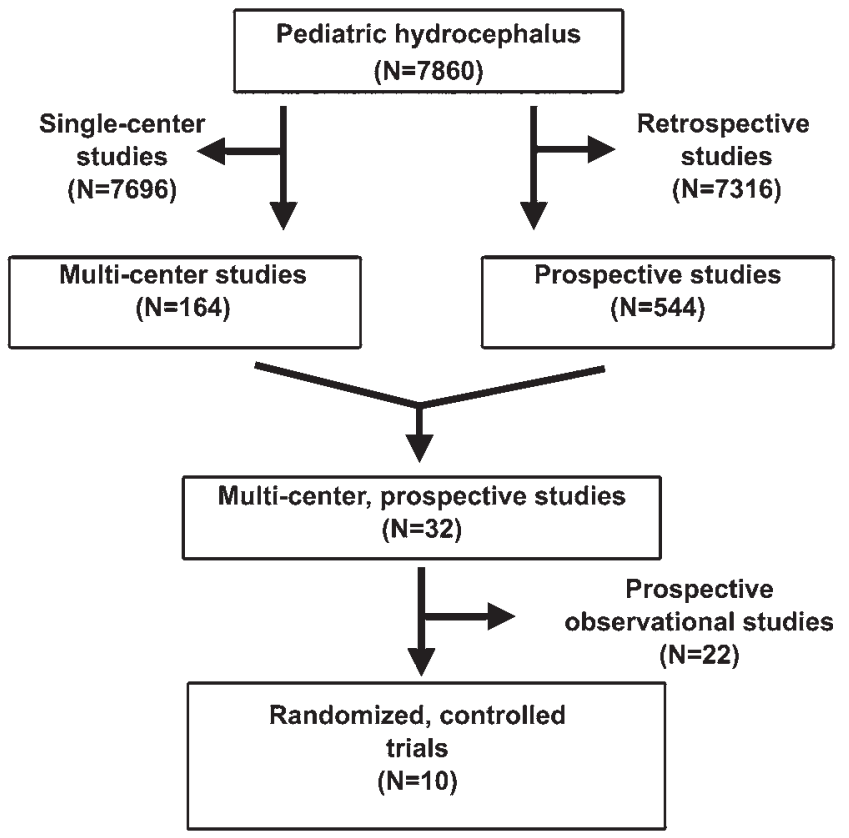

FIG. 1. Results of the review of the literature for multicenter prospective articles focused on pediatric hydrocephalus.

\section{Ventricular Catheter Placement}

Three multicenter prospective cohort studies have studied the accuracy of ventricular catheter placement. In 2003, Whitehead et al ${ }^{42}$ prospectively examined the use of ultrasound versus "conventional" ventricular catheter placement in first-time shunts across four HCRN centers. The use of ultrasound resulted in fewer catheters misplaced in the brain $(3 \%$ vs $22 \%, p=0.001)$ but did not significantly improve shunt survival $(\mathrm{p}=0.66)$. In 2017 , the same group conducted a quasi-prospective multicenter study ${ }^{41}$ by combining the raw data of the Shunt Design Trial (SDT), 7 the Endoscopic Shunt Insertion Trial (ESIT),${ }^{14}$ and the HCRN study described above. ${ }^{42}$ Variables such as the catheter's relationship with the choroid plexus or location of the catheter tip were not associated with shunt survival, but an anterior entry site significantly prevented shunt failure (hazard ratio [HR] $0.65,95 \%$ confidence interval $[\mathrm{CI}]$ 0.51-0.83). This was an unexpected finding and, given its easy modifiability, has spurred the HCRN to conduct an RCT comparing anterior and posterior ventricular catheter placement. ${ }^{43}$ Another group of three centers prospectively and sequentially examined standard ventricular catheter placement with anatomical landmarks and then electromagnetic navigation for firsttime shunt placement. ${ }^{10}$ Navigated shunts were more likely to be free floating in the CSF ( $\mathrm{p}=0.001)$ and had superior 30 -day survival $(\mathrm{p}=0.048)$. Unfortunately, the study had no adjustment for known confounders.

\section{Shunt Valve Design}

Although Hanlo et al. ${ }^{9}$ and Kestle and Walker ${ }^{17}$ published multicenter studies that were prospective, neither was technically a comparative effectiveness inquiry because all of the patients within the studies received the 
same valve, i.e., the Orbis Sigma Valve II (OSV II) and Strata, respectively. The OSV II study represented a mixed population of adult and pediatric cases while the Strata study was purely pediatric. Hanlo et al. ${ }^{9}$ reported that infants had higher OSV II failure on multivariable Cox regression analysis, but pediatric shunts as a whole did not fail significantly more than adult shunts. Kestle and Walker ${ }^{17}$ found a 1-year shunt survival rate of $67 \%(95 \%$ CI 60\%-74\%), which overlapped with the 1-year shunt survival rates found in the SDT for other valve types.

\section{Registries in Pediatric Hydrocephalus}

To our knowledge, there are two major pediatric hydrocephalus registries in the world: the United Kingdom Shunt Registry and the HCRN's Core Data Project (CDP).

\section{UK Shunt Registry}

The UK Shunt Registry, created in the 1990s, consists of a brief form and includes 78,000 procedures and 46,000 adult and pediatric patients. ${ }^{28}$ Using this database, Richards et al. ${ }^{32}$ published a multicenter prospective study with a matched-pair design comparing AICs with conventional catheters. There were 994 matched pairs $(\mathrm{n}=$ 1988) of adult and pediatric patients (42\%), and AICs were associated with a small but significant reduction in shunt infection $(3.0 \%$ vs $4.7 \%, \mathrm{p}=0.048)$.

\section{Hydrocephalus Clinical Research Network}

The HCRN's CDP is a hydrocephalus registry specific to pediatric hydrocephalus. The CDP represents 14 centers from Canada and the US, has a high degree of detail, and was created with 7 embedded prospective cohort studies. As of 2018, the CDP included 17,000 procedures and more than 8000 patients. ${ }^{33}$ The HCRN has published 6 prospective cohort studies, 5 of which were embedded in the original development of the CDP and 1 in tandem on $\mathrm{ETV}+\mathrm{CPC}$.

The first study by Kulkarni et al. ${ }^{18}$ was a hybrid study that compared a prospective cohort of first-time shunt patients from 7 centers with a historical cohort derived from the SDT and ESIT. The modern cohort of 1184 children demonstrated a lower failure rate (HR 0.82, 95\% CI 0.690.96) after adjusting for age and cause of hydrocephalus. This finding represented the first time the pediatric neurosurgery community has reported improved shunt survival rates (60\% vs $40 \%$ at 2 years) in the last few decades.

After this study, Simon et al. ${ }^{35}$ published a prospective cohort study from 6 HCRN centers representing 1036 children with first shunts and examined risk factors for shunt infection. The most striking finding, other than the risk factors of gastrostomy and age of 6-12 months, was that the best way to avoid shunt infection was to avoid shunt revision; patients with a single revision had a 3.9 times higher $\mathrm{HR}$ and those with $\geq 2$ revisions had a 13 times higher HR of shunt infection. A follow-up prospective cohort study from 7 centers within the HCRN found that the surgical treatment but not duration of intravenous antibiotics followed the 2004 Infectious Diseases Society of America guidelines. ${ }^{36}$ Given the importance of reducing shunt revision surgery, Riva-Cambrin et al. ${ }^{33}$ examined risk factors of shunt malfunction in a cohort of 1036 patients. In this study, age $<6$ months, a cardiac comorbidity, and endoscopic shunt placement were independent predictors of shunt failure. Cause of hydrocephalus, valve type, and valve programmability were not associated with shunt survival.

Embedded in the CDP, the HCRN has published the results of two multicenter prospective cohort studies on endoscopic treatment of hydrocephalus. Kulkarni et al. ${ }^{19}$ confirmed that the ETV Success Score $(p<0.001)$ and intraoperative visualization of a "naked" basilar artery ( $p$ $=0.023$ ) were predictors of ETV success. This study was also the first to publish prospectively collected complications for this procedure in a multicenter fashion, which remains the standard for future comparison. The newer $\mathrm{ETV}+\mathrm{CPC}$ procedure was examined prospectively in the CDP at 9 centers. ${ }^{20} \mathrm{~A}$ matched cohort of shunts had lower failure rates, and no difference in failure was found with a matched ETV cohort. They also confirmed the Ugandan data that a greater degree of CPC was associated with ETV+CPC success. ${ }^{38}$

\section{Strengths and Pitfalls of Registry-Based Multicenter Prospective Cohort Studies}

Most prospective cohort studies are quite expensive to organize and conduct, often similar to RCTs in both manhours and cost. Efficiency can be magnified by bundling multiple studies into a registry infrastructure. However, problems arise in funding. Most funders prefer to tie monies to specific studies and not to fund giant enterprises such as 10-year registries. Therefore, the registries discussed above have been supported by government funding (UK Shunt Registry) or philanthropy (HCRN).

\section{Prospective Multicenter Quality-Improvement Studies}

Two prospective multicenter quality-improvement studies have focused on pediatric hydrocephalus. Both are HCRN reports examining a shunt infection prevention protocol. In 2011, Kestle et al. ${ }^{16}$ reported a drop in shunt infection from $8.8 \%$ to $5.7 \%$ after protocol implementation at 4 institutions. A multivariable analysis found that BioGlide catheters and the use of antiseptic cream both increased the risk of shunt infection. The same group published a follow-up study in 2016 after altering the shunt protocol with the noted addition of AICs. ${ }^{15}$ This report of 1935 procedures at 8 centers demonstrated an infection rate of $6.0 \%(95 \%$ CI $5.1 \%-7.2 \%)$, which was not significantly different from the original protocol without AICs. These quality-improvement studies are unique and were designed to improve patient care and outcomes but have had the added benefit of improving knowledge of the surgical risk factors of shunt infection. ${ }^{16}$

\section{Discussion}

Our efforts in multicenter prospective hydrocephalus research are just scratching the surface. The results of many of the trials have been negative. This may be because we are in the infancy of studying hydrocephalus. There are many subtypes of hydrocephalus, and we know that etiology is one of the two most important prognostic 
factors in many hydrocephalus studies. Most studies so far have enrolled patients with hydrocephalus of a wide range of causes. This might explain the overall negative results, even in multicenter prospective trials with adequate sample size. This is analogous to performing studies to cure cancer by including patients with a variety of cancers. If we look at the pediatric oncology field, study protocols have entry criteria or stratification based on tumor histology, tumor stage, and now molecular markers. We are nowhere near this level of detail in hydrocephalus. We know that the etiologic subgroups, based on clinical findings, are important, but we need the same scrutiny of the disease categories with molecular markers and other factors that might indicate prognosis. We then need to conduct studies specific to those different conditions, and we may find that certain shunts or certain endoscopic techniques work in some subgroups but not others. We have already seen that $\mathrm{ETV}+\mathrm{CPC}$ has low success rates in the premature IVH population but much better rates in patients with aqueductal stenosis and myelomeningocele. Although some trials ${ }^{1,5,12,30,44}$ have incorporated etiology in their entry criteria, our tendency to group hydrocephalus as one condition in clinical trials must evolve and incorporate prognostic subgroups. In addition, the move toward patient-centered outcome is important. Many previous hydrocephalus trials have focused on device or procedure failure rather than developmental assessment. As discussed by Dr. Matson 65 years ago, ${ }^{26}$ growth and development of the child is the most important outcome. This requires prolonged followup of children treated in infancy, but is doable..$^{21,43}$

\section{Conclusions}

Hydrocephalus is the most common condition that we treat and our efforts to minimize its impact on children and families through prospective multicenter clinical research should continue to be among our highest priorities.

\section{References}

1. Adzick NS, Thom EA, Spong CY, Brock JW III, Burrows PK, Johnson MP, et al: A randomized trial of prenatal versus postnatal repair of myelomeningocele. N Engl J Med 364:993-1004, 2011

2. Bierbrauer KS, Storrs BB, McLone DG, Tomita T, Dauser R: A prospective, randomized study of shunt function and infections as a function of shunt placement. Pediatr Neurosurg 16:287-291, 1990-1991

3. Dan NG, Wade MJ: The incidence of epilepsy after ventricular shunting procedures. J Neurosurg 65:19-21, 1986

4. de Vries LS, Groenendaal F, Liem KD, Heep A, Brouwer AJ, van 't Verlaat $E$, et al: Treatment thresholds for intervention in posthaemorrhagic ventricular dilation: a randomised controlled trial. Arch Dis Child Fetal Neonatal Ed [epub ahead of print], 2018

5. de Vries LS, Liem KD, van Dijk K, Smit BJ, Sie L, Rademaker KJ, et al: Early versus late treatment of posthaemorrhagic ventricular dilatation: results of a retrospective study from five neonatal intensive care units in The Netherlands. Acta Paediatr 91:212-217, 2002

6. Dickerman RD, McConathy WJ, Morgan J, Stevens QE, Jolley JT, Schneider S, et al: Failure rate of frontal versus parietal approaches for proximal catheter placement in ventriculoperitoneal shunts: revisited. J Clin Neurosci 12:781-783, 2005
7. Drake JM, Kestle JR, Milner R, Cinalli G, Boop F, Piatt J Jr, et al: Randomized trial of cerebrospinal fluid shunt valve design in pediatric hydrocephalus. Neurosurgery 43:294-305, 1998

8. Etus V, Kahilogullari G, Karabagli H, Unlu A: Early endoscopic ventricular irrigation for the treatment of neonatal posthemorrhagic hydrocephalus: a feasible treatment option or not? A multicenter study. Turk Neurosurg 28:137-141, 2018

9. Hanlo PW, Cinalli G, Vandertop WP, Faber JA, Bøgeskov L, Børgesen SE, et al: Treatment of hydrocephalus determined by the European Orbis Sigma Valve II survey: a multicenter prospective 5-year shunt survival study in children and adults in whom a flow-regulating shunt was used. J Neurosurg 99:52-57, 2003

10. Hayhurst C, Beems T, Jenkinson MD, Byrne P, Clark S, Kandasamy J, et al: Effect of electromagnetic-navigated shunt placement on failure rates: a prospective multicenter study. J Neurosurg 113:1273-1278, 2010

11. Jenkinson MD, Gamble C, Hartley JC, Hickey H, Hughes D, Blundell M, et al: The British antibiotic and silver-impregnated catheters for ventriculoperitoneal shunts multi-centre randomised controlled trial (the BASICS trial): study protocol. Trials 15:4, 2014

12. Kennedy CR, Ayers S, Campbell MJ, Elbourne D, Hope P, Johnson A: Randomized, controlled trial of acetazolamide and furosemide in posthemorrhagic ventricular dilation in infancy: follow-up at 1 year. Pediatrics 108:597-607, 2001

13. Kestle J, Drake J, Milner R, Sainte-Rose C, Cinalli G, Boop F, et al: Long-term follow-up data from the Shunt Design Trial. Pediatr Neurosurg 33:230-236, 2000

14. Kestle JR, Drake JM, Cochrane DD, Milner R, Walker ML, Abbott R III, et al: Lack of benefit of endoscopic ventriculoperitoneal shunt insertion: a multicenter randomized trial. J Neurosurg 98:284-290, 2003

15. Kestle JR, Holubkov R, Douglas Cochrane D, Kulkarni AV, Limbrick DD Jr, Luerssen TG, et al: A new Hydrocephalus Clinical Research Network protocol to reduce cerebrospinal fluid shunt infection. J Neurosurg Pediatr 17:391-396, 2016

16. Kestle JR, Riva-Cambrin J, Wellons JC III, Kulkarni AV, Whitehead WE, Walker ML, et al: A standardized protocol to reduce cerebrospinal fluid shunt infection: the Hydrocephalus Clinical Research Network Quality Improvement Initiative. J Neurosurg Pediatr 8:22-29, 2011

17. Kestle JR, Walker ML: A multicenter prospective cohort study of the Strata valve for the management of hydrocephalus in pediatric patients. J Neurosurg 102 (2 Suppl):141-145, 2005

18. Kulkarni AV, Riva-Cambrin J, Butler J, Browd SR, Drake JM, Holubkov R, et al: Outcomes of CSF shunting in children: comparison of Hydrocephalus Clinical Research Network cohort with historical controls: clinical article. J Neurosurg Pediatr 12:334-338, 2013

19. Kulkarni AV, Riva-Cambrin J, Holubkov R, Browd SR, Cochrane DD, Drake JM, et al: Endoscopic third ventriculostomy in children: prospective, multicenter results from the Hydrocephalus Clinical Research Network. J Neurosurg Pediatr 18:423-429, 2016

20. Kulkarni AV, Riva-Cambrin J, Rozzelle CJ, Naftel RP, Alvey JS, Reeder RW, et al: Endoscopic third ventriculostomy and choroid plexus cauterization in infant hydrocephalus: a prospective study by the Hydrocephalus Clinical Research Network. J Neurosurg Pediatr 21:214-223, 2018

21. Kulkarni AV, Schiff SJ, Mbabazi-Kabachelor E, Mugamba J, Ssenyonga P, Donnelly R, et al: Endoscopic treatment versus shunting for infant hydrocephalus in Uganda. N Engl J Med 377:2456-2464, 2017

22. Kulkarni AV, Sgouros S, Constantini S: International Infant Hydrocephalus Study: initial results of a prospective, multicenter comparison of endoscopic third ventriculostomy 
(ETV) and shunt for infant hydrocephalus. Childs Nerv Syst 32:1039-1048, 2016

23. Leggate JL, Baxter P, Minns RA, Steers AJ: Epilepsy following ventricular shunt placement. J Neurosurg 68:318-319, 1988

24. Lind CR, Correia JA, Law AJ, Kejriwal R: A survey of surgical techniques for catheterising the cerebral lateral ventricles. J Clin Neurosci 15:886-890, 2008

25. Lind CR, Tsai AM, Lind CJ, Law AJ: Ventricular catheter placement accuracy in non-stereotactic shunt surgery for hydrocephalus. J Clin Neurosci 16:918-920, 2009

26. Matson DD: Hydrocephalus treated by arachnoid-ureterostomy; report of 50 cases. Pediatrics 12:326-334, 1953

27. National Institutes of Health: Single IRB policy for multi-site research. NIH.gov (https://grants.nih.gov/policy/clinicaltrials/single-irb-policy-multi-site-research.htm) [Accessed October 11, 2018]

28. O'Kane MC, Richards H, Winfield P, Pickard JD: The United Kingdom Shunt Registry. Eur J Pediatr Surg 7 (Suppl 1):56, 1997

29. Pollack IF, Albright AL, Adelson PD: A randomized, controlled study of a programmable shunt valve versus a conventional valve for patients with hydrocephalus. Neurosurgery 45:1399-1411, 1999

30. Ventriculomegaly Trial Group: Randomised trial of early tapping in neonatal posthaemorrhagic ventricular dilatation. Arch Dis Child 65:3-10, 1990

31. Ravindra V, Kestle J: Writing a clinical research question. Neurosurgery [in press], 2018

32. Richards HK, Seeley HM, Pickard JD: Efficacy of antibioticimpregnated shunt catheters in reducing shunt infection: data from the United Kingdom Shunt Registry. J Neurosurg Pediatr 4:389-393, 2009

33. Riva-Cambrin J, Kestle JR, Holubkov R, Butler J, Kulkarni AV, Drake J, et al: Risk factors for shunt malfunction in pediatric hydrocephalus: a multicenter prospective cohort study. J Neurosurg Pediatr 17:382-390, 2016

34. Schulz M, Bührer C, Pohl-Schickinger A, Haberl H, Thomale UW: Neuroendoscopic lavage for the treatment of intraventricular hemorrhage and hydrocephalus in neonates. J Neurosurg Pediatr 13:626-635, 2014

35. Simon TD, Butler J, Whitlock KB, Browd SR, Holubkov R, Kestle JR, et al: Risk factors for first cerebrospinal fluid shunt infection: findings from a multi-center prospective cohort study. J Pediatr 164:1462-1468.e2, 2014

36. Simon TD, Kronman MP, Whitlock KB, Gove N, Browd SR, Holubkov R, et al: Variability in management of first cerebrospinal fluid shunt infection: a prospective multi-institutional observational cohort study. J Pediatr 179:185-191.e2, 2016

37. Tulipan N, Wellons JC III, Thom EA, Gupta N, Sutton LN, Burrows PK, et al: Prenatal surgery for myelomeningocele and the need for cerebrospinal fluid shunt placement. J Neurosurg Pediatr 16:613-620, 2015

38. Warf BC, Mugamba J, Kulkarni AV: Endoscopic third ventriculostomy in the treatment of childhood hydrocephalus in
Uganda: report of a scoring system that predicts success. J Neurosurg Pediatr 5:143-148, 2010

39. Wellons JC III, Shannon CN, Holubkov R, Riva-Cambrin J, Kulkarni AV, Limbrick DD Jr, et al: Shunting outcomes in posthemorrhagic hydrocephalus: results of a Hydrocephalus Clinical Research Network prospective cohort study. J Neurosurg Pediatr 20:19-29, 2017

40. Wellons JC III, Shannon CN, Kulkarni AV, Simon TD, RivaCambrin J, Whitehead WE, et al: A multicenter retrospective comparison of conversion from temporary to permanent cerebrospinal fluid diversion in very low birth weight infants with posthemorrhagic hydrocephalus. J Neurosurg Pediatr 4:50-55, 2009

41. Whitehead WE, Riva-Cambrin J, Kulkarni AV, Wellons JC III, Rozzelle CJ, Tamber MS, et al: Ventricular catheter entry site and not catheter tip location predicts shunt survival: a secondary analysis of 3 large pediatric hydrocephalus studies. J Neurosurg Pediatr 19:157-167, 2017

42. Whitehead WE, Riva-Cambrin J, Wellons JC III, Kulkarni AV, Holubkov R, Illner A, et al: No significant improvement in the rate of accurate ventricular catheter location using ultrasound-guided CSF shunt insertion: a prospective, controlled study by the Hydrocephalus Clinical Research Network. J Neurosurg Pediatr 12:565-574, 2013

43. Whitelaw A, Evans D, Carter M, Thoresen M, Wroblewska J, Mandera M, et al: Randomized clinical trial of prevention of hydrocephalus after intraventricular hemorrhage in preterm infants: brain-washing versus tapping fluid. Pediatrics 119:e1071-e1078, 2007

44. Whitelaw A, Jary S, Kmita G, Wroblewska J, Musialik-Swietlinska E, Mandera M, et al: Randomized trial of drainage, irrigation and fibrinolytic therapy for premature infants with posthemorrhagic ventricular dilatation: developmental outcome at 2 years. Pediatrics 125:e852-e858, 2010

\section{Disclosures}

The authors report no conflict of interest concerning the materials or methods used in this study or the findings specified in this paper.

\section{Author Contributions}

Conception and design: both authors. Acquisition of data: both authors. Analysis and interpretation of data: both authors. Drafting the article: both authors. Critically revising the article: both authors. Reviewed submitted version of manuscript: both authors. Approved the final version of the manuscript on behalf of both authors: Kestle.

\section{Correspondence}

John R. W. Kestle: Primary Children's Hospital, University of Utah, Salt Lake City, UT. neuropub@hsc.utah.edu. 